\title{
Nicotine exposure and bronchial epithelial cell nicotinic acetylcholine receptor expression in the pathogenesis of lung cancer
}

\author{
John D. Minna \\ Hamon Center for Therapeutic Oncology Research and Departments of Internal Medicine and Pharmacology, University of Texas Southwestern \\ Medical Center, Dallas, Texas, USA
}

J. Clin. Invest. 111:31-33 (2003). doi:10.1172/JCI200317492.

\begin{abstract}
Forty-eight million Americans smoke because of addiction to nicotine, and prevention of smoking initiation and new methods for aiding in smoking cessation are some of the most important opportunities for disease prevention available (1). This smoking addiction results from nicotine acting on neuronal nicotinic acetylcholine receptors (nAchRs) in the brain in key regions controlling behavior. There is detailed structural and functional information on neuronal nAchRs that are the prototype of ligand-gated ion channels mediating transmission of endogenous acetylcholine (Ach) and exogenous nicotine signals in the central and peripheral nervous system (2). In addition to the well-formulated role of nAchRs in the etiology of addiction, emerging evidence suggests that nicotine contributes directly to lung carcinogenesis through stimulation of nAchRs in non-neuronal cells. Our
\end{abstract}

\footnotetext{
Address correspondence to: John D. Minna, Hamon Center for Therapeutic Oncology Research, NB8.206, University of Texas Southwestern Medical Center, 6000 Harry Hines Boulevard, Dallas, Texas 75390-8593, USA. Phone: (214) 648-4900;

Fax: (214) 648-4940;

E-mail: john.minna@utsouthwestern.edu.

Conflict of interest: The author has declared that no conflict of interest exists.

Nonstandard abbreviations used: nicotinic acetylcholine receptor (nAchR); acetylcholine (Ach); N'Nitrosonornicotine (NNN);

4(methylnitrosamino)-1-(3-pyridyl)-1-

butanone (NNK); normal human bronchial epithelial cell (NHBE); small airway epithelial cell (SAEC); protein kinase B (PKB); B cell lymphoma 2 (BLC2); trans-retinoic acid (RA); retinoic acid receptor (RAR); Janus kinase 2 (JAK2); mitogen-activated protein kinase (ERK); retinoid X receptor (RXR).
}

group showed that many lung cancers expressed nAchRs and that low concentrations of nicotine blocked the induction of apoptosis in these cells (3). Conti-Fine's group demonstrated that functional nAchRs are expressed in human and rodent bronchial epithelial cells $(4,5)$. While not in the bronchial epithelium, Grando and colleagues showed that human epidermal keratinocytes synthesize, secrete, and degrade Ach which is used to mediate stimulation of calcium influx and cell differentiation through $\alpha 7 \mathrm{nAch}$ Rs on these same cells $(6,7)$. nAchRs bind not only nicotine, but also nicotine-derived nitrosamines such as the tobacco specific carcinogens $\mathrm{N}^{\prime}$-Nitrosonornicotine (NNN) and 4(methylnitrosamino)1-(3-pyridyl)-1-butanone (NNK), which are potent lung carcinogens. There is evidence from Schuller's group that lung cancer and fetal pulmonary neuroendocrine cells express nAchRs that bind these ligands with higher affinity than nicotine stimulating the growth of these cells (8-10).

Nicotine and NNK activate Akt via nAchRs in bronchial epithelial cells In this issue of the JCI, Brognard and colleagues have extended these results by presenting intriguing findings concerning the activation of the Akt signaling pathway by nicotine and NNK in cultured lung epithelial cells (11). Using short-term cultures of large (normal human bronchial epithelial cells [NHBEs]), and small airway epithelial cells (SAECs) they showed that stimulation with nicotine and NNK in pharmacologically relevant concentrations (in the $100 \mathrm{nM}$ range which is attained in the plasma of smokers) induced phosphorylation of Akt on physiologically relevant serine and threonine residues within minutes. In addition, increased Akt phosphorylation was detected in vivo in the lungs of NNK treated mice and in human lung cancers arising in smokers (11). Akt located at $14 \mathrm{q} 32.3$ in humans and sometimes called protein kinase B ([PKB] Online Mendelian Inheritance in Man no. 164730) is a multifunctional serine-threonine protein kinase that was initially identified as a mouse retrovirus oncogene in

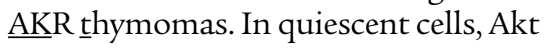
resides in the cytosol in a low-activity conformation. Upon cellular stimulation, Akt is activated through recruitment to cellular membranes by PI3K lipid products and by phosphorylation by $3^{\prime}$ phosphoinositide-dependent kinase-1 resulting in the activation of a variety of downstream targets including those of the NF- $\mathrm{KB}$ pathway (12). Thus, Akt activation plays a key role in inhibiting apoptosis in multiple tissues in response to growth factor deprivation or oncogene stimulation. It follows that Akt and its pathway are major targets for new cancer drug discovery.

\section{nAchR subtype, target cell differences, and other mechanisms by which nicotine could block apoptosis}

Brognard et al. found the expression of nAchR subunit types, timing and duration of the Akt response, and effects of Akt phosphorylation of downstream substrates varied between NHBEs and SAECs following stimula- 
tion with nicotine or NNK (11). Specific $\mathrm{nAch}$ antagonists were used to show that the $\alpha 3$ or $\alpha 4$ subtypes of nAchRs mediated the nicotine effect, while $\alpha 7$-containing $n A c h R s$ mediated the NNK effect. This finding suggests that the mechanisms and results of activation of Akt by nicotine and NNK may be slightly different. Nicotine also caused a loss of contact inhibition at high cell densities in culture and when lung epithelial cells were challenged with a variety of apoptotic inducing stimuli, nicotine and NNK inhibited the induction of apoptosis (11). It will also be important to know if nicotine can suppress apoptosis in epithelial cells engendered by the expression of activated oncogenes such as mutated Ras or over expressed c-myc. Other studies have shown that nicotine acting through nAchRs also upregulate functional nAchRs providing a positive feedback loop that could amplify nicotine responses on epithelial cells, so it will be important to know if nicotine exposure from smoking upregulates $n A c h R s$ in the bronchial epithelium (5). While the current report focuses on Akt activation, others have shown that nicotine stimulates the phosphorylation of key residues in the anti-apoptotic protein B cell lymphoma gene 2 (BCL2) through activation of protein kinase $C \alpha$ and phospholipase $C$ to inhibit apoptosis (13). The failure of retinoids to prevent lung cancer development in smokers has been a great disappointment. Thus, it is also of interest to learn that nicotine suppresses the growth inhibitory effects of trans-retinoic acid (RA) on lung cancer cells by inhibiting retinoic acid receptor (RAR) $\beta$ expression by inducing expression of an orphan RA receptor TR3, while inhibition of lung cancer cells by retinoid $X$ receptorselective (RXR-selective) retinoids was unaffected by nicotine (14). Taken together, these findings suggest the hypothesis that nicotine or carcinogenic derivatives of nicotine acting through specific nAchRs expressed in lung epithelial cells play a key role in the pathogenesis of lung cancer, through loss of contact inhibition and development of resistance to apoptosis (11). Thus, nicotine and NNK could act as tumor promoters to facilitate the outgrowth of cells with genetic damage, while NNK, could also provide this genotoxic damage.

\section{Further questions: cellular targets and the role of other $n A c h R$ subunit types}

As with every good study, the report by Brognard et al. (11) suggests a variety of questions and further experiments. Which cells in the bronchial epithelium express nAchRs and which of these cells develop activated Akt? What is the distribution of $\mathrm{nAch} R$ expression in other epithelial tissues throughout the body and does this expression correlate with the propensity of these tissues to undergo tobacco related carcinogenic changes? In the current study, $\alpha 3$ and $\alpha 4$ nAchR subunits mediated Akt effects by nicotine while $\alpha 7$ mediated the effects by NNK. Nevertheless, other nAchR subtypes were also expressed in the epithelial cells and we need to know if they modulate effects important to cell growth and survival. In the current study the effects on cell growth and apoptosis inhibition were statistically significant but quantitatively small. Thus, we need to know if there is a subpopulation of cells, such as the basal cells of the bronchial epithelium, where nicotine and nAchRs have quantitatively greater effects on these processes.

\section{Required patient-oriented research}

What is the timing of this activation by cigarette smoking in vivo? What about people who have quit smoking and the effects of passive exposure to cigarette smoke and nicotine? We will want to test bronchial epithelium for Akt activation of current and former smokers, smokers abstaining from nicotine for several hours, persons quitting smoking but on nicotine patches, and people exposed to environmental tobacco smoke. Since many lung cancers appear to have constitutive Akt activation we will want to know how and when this sustained activation take place (15). By contrast, we need to know if in some patients' tumors the system is still responsive such that nicotine delivered by continued smoking may also contribute to tumor progression, metastasis, or interfere with anti-cancer therapies seeking to induce tumor apoptosis. Thus, it would be very instructive to sample tumors in patients before and after a smoking nicotine challenge to see if the Akt in tumors was responsive to this signaling pathway in vivo. Are there polymorphisms in nAchRs or in nicotine metabolizing enzymes that influence this activation providing genetic and racial variation in Akt activation by nicotine and potentially the development of lung cancer (16)? In the US approximately $15 \%$ of lung cancers arise in lifetime never-smokers, while $50 \%$ of all lung cancers arise in persons who have quit smoking for at least 5 years. What is the status of nAchRs, nicotine responsiveness, and Akt signaling in the bronchial epithelium and lung tumors of these patients who are not recently exposed to nicotine? While cigarette smoke supplies nicotine there is considerable information on the synthesis of Ach in non-neuronal cells raising the question of whether these endogenous sources of Ach play a role similar to nicotine in tumor development (17).

\section{Understanding the biochemical events generated after nicotine interacts with its receptor}

The biochemical events between nicotine interacting with nAchRs leading to activation of Akt remain to be researched. In neuronal systems of neuroprotection against apoptosis induction, it appears that nicotine stimulation of $\alpha 7 \mathrm{nAchR}$ transduces signals in a cascade to phosphatidylinositol-3-kinase and Akt via Janus kinase 2 (JAK2) (18). Other studies provide evidence for a novel signaling route coupling the stimulation of $\alpha 7$ $n$ AchR to the activation of mitogenactivated protein kinases ERK1/2, in a $\mathrm{Ca}^{2+}$ and protein kinase A-dependent manner (19). Once the pathway is understood we will want to know if alterations in this pathway independent of nicotine exposure lead to constitutive activation of Akt in tumors. Knockout mouse models targeting nAchRs will allow one to test the hypothesis that such knockout models should be resistant to NNKinduced carcinogenesis (7).

\section{Nicotine and angiogenesis}

In addition to the effects on bronchial epithelium, there is a growing literature on the role of nicotine and endogenous cholinergic pathways acting through $\mathrm{nAchRs}$ (predominantly through $\alpha 7$ nAchRs) on endothelial cell growth and angiogenesis including tumor angiogenesis $(20,21)$. Such a nAchR-induced endothelial network formation was partially dependent on 
VEGF, was completely dependent on the phosphatidylinositol 3-kinase and mitogen-activated protein kinase pathways, and finally resulted in NF- $\kappa B$ activation (21). The common metabolite of nicotine, cotinine, which presumably should be inactive, also needs to be tested in these assays since other studies have shown that both nicotine and cotinine upregulate VEGF expression and potentially other angiogenic growth factors in endothelial cells (22). Given the production of VEGF by many tumor cells, one can envisage that the effect of nicotine or endogenous Ach on tumor cells could also use similar signaling pathways for growth and protection against apoptosis. Thus, nicotine could play an additional role in carcinogenesis through being a pro-angiogenic agent. Finally, manipulating this system to prevent or treat cancer will require careful thought. For example, in some situations nicotine acting through $\mathrm{nAch}$ s can stimulate wound healing by promoting angiogenesis while in other systems nicotine acting through nAchRs appears to inhibit kerotinocyte migration and wound healing $(23,24)$.

\section{Acknowledgments}

This work was supported by grant NCI P50CA70907 from the Lung Cancer Special Program of Research Excellence (SPORE).

1. Karnath, B. 2002. Smoking cessation. Am. J. Med. 112:399-405.
2. Itier, V., and Bertrand, D. 2001. Neuronal nicotinic receptors: from protein structure to function. FEBS Lett. 504:118-125.

3. Maneckjee, R., and Minna, J.D. 1994. Opioids induce while nicotine suppresses apoptosis in human lung cancer cells. Cell Growth Differ. 5:1033-1040.

4. Maus, A.D., et al. 1998. Human and rodent bronchial epithelial cells express functional nicotinic acetylcholine receptors. Mol. Pharmacol. 54:779-788.

5. Wang, Y., et al. 2001. Human bronchial epithelial and endothelial cells express alpha7 nicotinic acetylcholine receptors. Mol. Pharmacol. 60:1201-1209.

6. Grando, S.A., et al. 1996. Activation of keratinocyte nicotinic cholinergic receptors stimulates calcium influx and enhances cell differentiation. J. Invest. Dermatol. 107:412-418.

7. Arredondo, J., et al. 2002. Central role of alpha7 nicotinic receptor in differentiation of the stratified squamous epithelium. J. Cell. Biol. 159:325-336

8. Schuller, H.M., and Orloff, M. 1998. Tobacco-specific carcinogenic nitrosamines. Ligands for nicotinic acetylcholine receptors in human lung cancer cells. Biochem. Pharmacol. 55:1377-1384.

9. Plummer, H.K., 3rd, Sheppard, B.J., and Schuller, H.M. 2000. Interaction of tobacco-specific toxi cants with nicotinic cholinergic regulation of fetal pulmonary neuroendocrine cells: implications for pediatric lung disease. Exp. Lung. Res. 26:121-135.

10. Schuller, H.M., Jull, B.A., Sheppard, B.J., and Plummer, H.K. 2000. Interaction of tobacco-specific toxicants with the neuronal alpha (7) nicotinic acetylcholine receptor and its associated mitogenic signal transduction pathway: potential role in lung carcinogenesis and pediatric lung disorders. Eur. J. Pharmacol. 393:265-277.

11. West, K.A., et al. 2003. Rapid Akt activation by nicotine and a tobacco carcinogen modulates the phenotype of normal human airway epithelial cells. J. Clin. Invest. 111:81-90. doi:10.1172/JCI200316147.

12. Vanhaesebroeck, B., and Alessi, D.R. 2000. The PI3K-PDK1 connection: more than just a road to PKB. Biochem. J. 346:561-576.

13. Mai, H., May, W.S., Gao, F., Jin, Z., and Deng, X 2002. A functional role for nicotine in $\mathrm{Bcl} 2$ phosphorylation and suppression of apoptosis. J. Biol.
Chem. doi:10.1074/jbc.M209044200.

14. Chen, G.Q., Lin, B., Dawson, M.I., and Zhang, X.K. 2002. Nicotine modulates the effects of retinoids on growth inhibition and RAR beta expression in lung cancer cells. Int. J. Cancer. 99:171-178.

15. Brognard, J., Clark, A.S., Ni, Y., and Dennis, P.A 2001. Akt/protein kinase B is constitutively active in non-small cell lung cancer cells and promotes cellular survival and resistance to chemotherapy and radiation. Cancer Res. 61:3986-3997.

16. Benowitz, N.L., Perez-Stable, E.J., Herrera, B., and Jacob, P., 3rd. 2002. Slower metabolism and reduced intake of nicotine from cigarette smok ing in Chinese-Americans. J. Natl. Cancer Inst. 94:108-115

17. Conti-Fine, B.M., Navaneetham, D., Lei, S., and Maus, A.D. 2000. Neuronal nicotinic receptors in non-neuronal cells: new mediators of tobacco toxicity? Eur. J. Pharmacol. 393:279-294.

18. Shaw, S., Bencherif, M., and Marrero, M.B. 2002. Janus kinase 2: an early target of Alpha7-mediated nicotinic acetylcholine receptor neuroprotection against Abeta (1-42) Amyloid. J. Biol. Chem. 277:44920-44924.

19. Dajas-Bailador, F.A., Soliakov, L., and Wonnacott, S. 2002. Nicotine activates the extracellular signal-regulated kinase $1 / 2$ via the alpha7 nicotinic acetylcholine receptor and protein kinase $\mathrm{A}$, in $\mathrm{SH}-\mathrm{SY} 5 \mathrm{Y}$ cells and hippocampal neurones. J. Neurochem. 80:520-530.

20. Heeschen, C., et al. 2001. Nicotine stimulates angiogenesis and promotes tumor growth and atherosclerosis. Nat. Med. 7:833-839.

21. Heeschen, C., Weis, M., Aicher, A., Dimmeler, S. and Cooke, J.P. 2002. A novel angiogenic pathway mediated by non-neuronal nicotinic acetylcholine receptors. J. Clin. Invest. 110:527-536. doi:10.1172/JCI200214676

22. Conklin, B.S., Zhao, W., Zhong, D.S., and Chen, C. 2002. Nicotine and cotinine up-regulate vascular endothelial growth factor expression in endothelial cells. Am. J. Pathol. 160:413-418.

23. Jacobi, J., et al. 2002. Nicotine accelerates angiogenesis and wound healing in genetically diabetic mice. Am. J. Pathol. 161:97-104.

24. Zia, S., Ndoye, A., Lee, T.X., Webber, R.J., and Grando, S.A. 2000. Receptor-mediated inhibition of keratinocyte migration by nicotine involves modulations of calcium influx and intracellular concentration. J. Pharmacol. Exp. Ther. 293:973-981. 